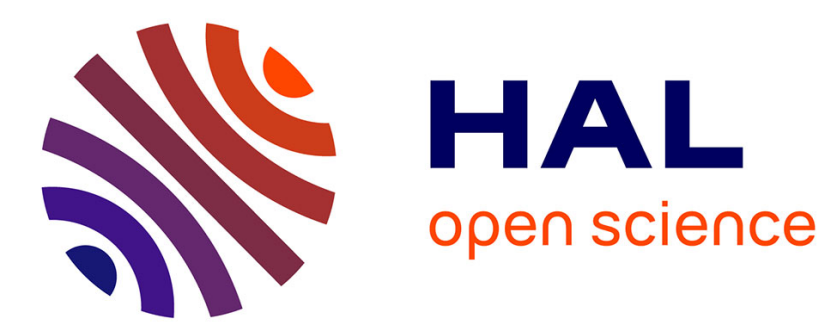

\title{
Prime numbers as a tool to design distributed algorithms
}

Michel Raynal

\section{To cite this version:}

Michel Raynal. Prime numbers as a tool to design distributed algorithms. [Research Report] RR-1001, INRIA. 1989. inria-00075558

\section{HAL Id: inria-00075558 \\ https://hal.inria.fr/inria-00075558}

Submitted on 24 May 2006

HAL is a multi-disciplinary open access archive for the deposit and dissemination of scientific research documents, whether they are published or not. The documents may come from teaching and research institutions in France or abroad, or from public or private research centers.
L'archive ouverte pluridisciplinaire HAL, est destinée au dépôt et à la diffusion de documents scientifiques de niveau recherche, publiés ou non, émanant des établissements d'enseignement et de recherche français ou étrangers, des laboratoires publics ou privés. 


\section{Rapports de Recherche}

Q4) 1. V. A

จ 54 (4)

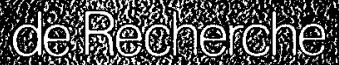

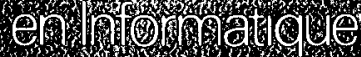

-t - a

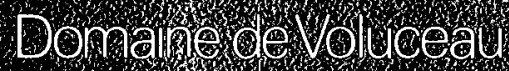
Rogr.

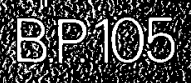

78153 - - - ashay Cecex 6.

Tò (4)

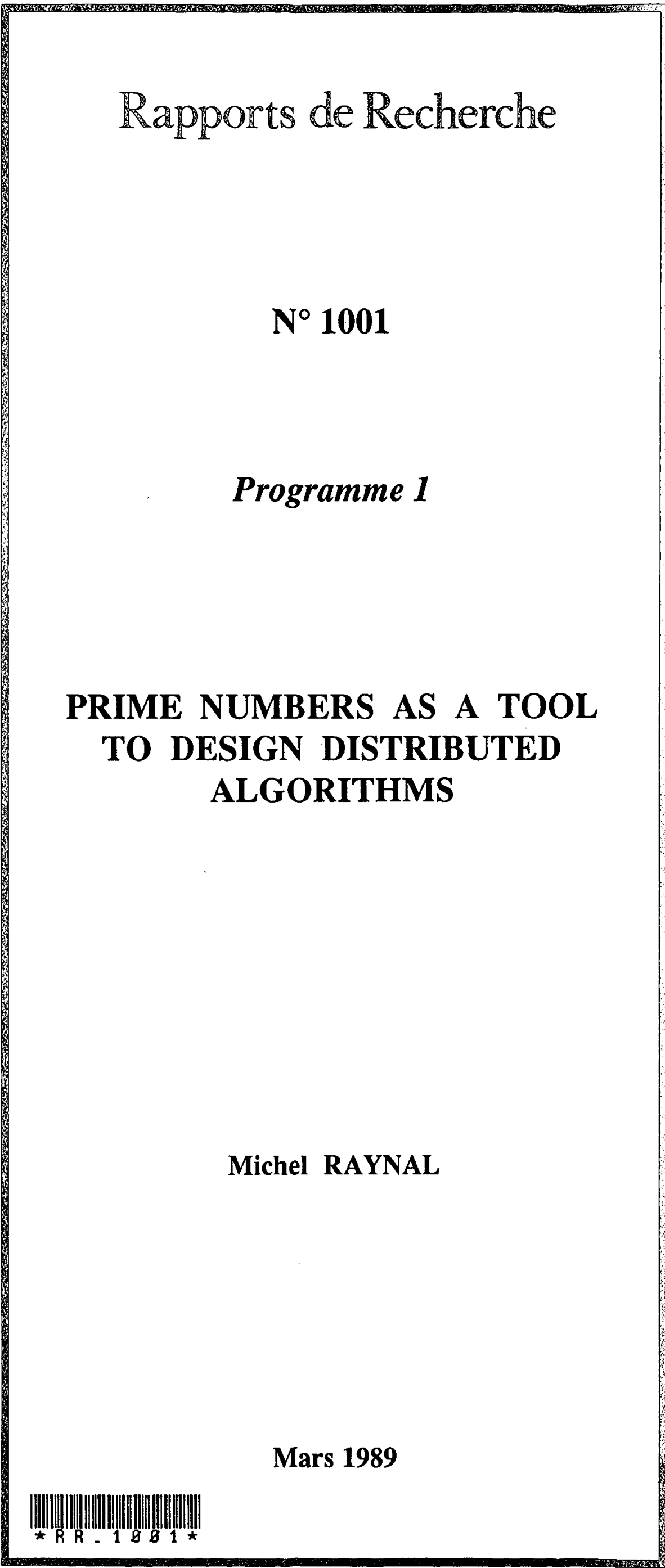




\title{
PRIME NUMBERS AS A TOOL TO DESIGN DISTRIBUTED ALGORITHMS
}

Les nombres premiers : un outil pour la conception des algorithms répartis

\author{
Publication Interne $\mathrm{n}^{\circ} 458$ - 14 Pages - Février 1989
}

\author{
Michel RAYNAL
}

\author{
IRISA \\ Campus de Bcaulicu \\ 35042 Rennes Cedcx \\ - FRANCE - \\ E-mail: raynal@irisa.fr
}

\begin{abstract}
Prime numbers are investigated as a tool to design distributed control algorithms. One of major drawbacks in designing such algorithms lies in the inability for onc or scveral processes (or even for an external observer) to catch instantancously some part of the global state of the system. It is shown in this paper how, in some cases, prime numbers can be used to make distributed observalions allowing to make consistent decisions. Two very different distributed algorithms are produced as an illustration of the proposed tool (a mutual exclusion and a tcrmination detection algorithms).
\end{abstract}

Résumé :

L'impossibilité d'obtenir un état global de manière instantanée constitue l'un des problèmes les plus délicats à résoudre lors de la conception d'un algorithme de contrôle réparti. Des outils algorithmiques particuliers tels que les horloges logiques ou les marqueurs ont été introduit afin de pallier cette incapacité. On propose ici un autre outil pour résoudre ces problèmes : les nombres premiers. Deux algorithms distribués (l'un réalisant une exclusion mutuelle, l'autre détectant la terminaison répartie) sont exhibés pour illustrer l'outil proposé. 


\title{
PRIME NUMBERS AS A TOOL TO DESIGN DISTRIBUTED ALGORITHMS
}

\author{
Michel RAYNAL
}

\author{
IRISA \\ Campus de Beaulieu \\ 35042 Rennes Cedex \\ - FRANCE - \\ E-mail: raynal@irisa.fr
}

\begin{abstract}
Prime numbers are investigated as a tool to design distributed control algorithms. One of major drawbacks in designing such algorithms lies in the inability for one or several processes (or even for an extcrnal observer) to catch instantaneously some part of the global state of the system. It is shown in this paper how, in some cases, prime numbers can be used to make distributed observations allowing to make consistent decisions. Two very different distributed algorithms are produced as an illustration of the proposed tool (a mutual exclusion and a termination detection algorithms).
\end{abstract}

\section{INTRODUCTION}

One of the more basic problems found in asynchronous distributed computations and distributed systems lies in the inability for one or several processes the system is made of (or even for an observer external to the system) to take instantaneously a consistent global snapshot[LEL77, FRA80, CL85, RAY88]. Among others causes, this inability comes from the not-bounded communication delays for messages. So when designing a distributed algorithm needing some kind of global knowledge, one has to introduce some devices allowing to get a consistent view of the part of the system he needs. Logical clocks introduced by Lamport [LAM78] to distribute 
a state machine or markers introduced by Misra [MIS83] and used on fifo channels by Chandy and Lamport [CL85] are such devices.

This paper studies another such a device: prime numbers; each process of the system is endowed with a (different) prime number. As we will see the unicity of the decomposition of an integer in a product of prime numbers is a property which allows one or several processes (or an external observer) to take consistent decisions concerning some aspects of the global state of the system.

The $\S 2$ states the hypotheses. Then two simple and elegant distributed control algorithms are produced: a mutual exclusion algorithm $(\S 3)$ and a termination detection algorithm ( $\$ 4)$. Mutual exclusion and termination detection are two paradigms of distributed control. Our aim is not to obtain efficient algorithms from a number of messages point of view but to investigate and to show that prime numbers and their properties can be a useful tool when designing some kind of algorithms in a distributed context.

\section{HYPOTHESES}

We consider a distributed system made of $n$ sites: $P_{0}, P_{1}, . ., P_{n-1}$; one and only one process is associated to each site. These processes are linked by a connection network; there is no central memory and processes communicate solely by means of messages with unpredictable (and non-zero) transmission delays; communication channels are bidirectionnal.

Each process $P_{i}$ is endowed with an attribute $a_{i}$; these attributes are natural integers, different from 1, and prime two by two.

\section{A MUTUAL EXCLUSION ALGORITHM}

\subsection{The algorithm}

The network is supposed totally connected. Each $P_{i}$ owns a variable $x_{i}$ initialized to $a_{i}$ except for one $x_{k}$ initialized to 1 . Each process $P_{i}$ can read any variable $x_{j}$. (This can be easily implemented by a request message from $P_{i}$ to $P_{j}$ followed by a reply message from $P_{j}$ to $P_{i}$ ).

$$
\text { Let } Q \text { be } \prod_{i=1}^{n} a_{i}
$$

Protocols to enter and exit the critical section are the following ones for each $P_{i}$ : 


$$
\begin{aligned}
& \text { Wait } \prod_{m=1}^{n}\left(x_{m}=Q / a_{i}\right) \\
& <\text { critical section }>\text {; } \\
& x_{i}:=x_{i} * a_{i} / a_{j}
\end{aligned}
$$

In the exit part of the protocol the effect of the multiplication by $a_{i}$ is the loss by $P_{i}$ of the privilege to use the critical section; the effect of the division by $a_{j}$ is to give $P_{j}$ the privilege. If $j=(i+1) \bmod n$ the privilege to use the critical section turns around the logical ring: $P_{k}, P_{k+1}, . ., P_{0}, P_{1}, . ., P_{k}$, as in [LEL77, DIJ74].

Remark: The wait operation is implemented by having $P_{i}$ to ask the $P_{j} s$ the values of their variables $x_{j}$ from time to time until the condition becomes true.

\subsection{Proof}

The privilege to enter the critical section is expressed by some configuration of the state variables $x_{i}$. It is very easy to see that initially only the process $P_{k}$ (the one with $x_{k}$ initialized to 1 ) can enter the critical section (without loss of generality we suppose $k=0$ ).

It is easy to show that in a centralized context (the $x_{i}$ are stored in a central memory and the readings get always the last values of the variables) the privilege turns around the logical ring iff $a_{i} \neq a_{j}, \forall i \neq j$.

In a distributed context a problem comes from the arbitrary (but finite) delays of the request and reply messages. Two processes $P_{j}$ and $P_{k}$ can use at the same time for the value of some $x_{i}$, two different values the variable $x_{i}$ had previously. Consequently we have to prove that, despite these potentially mutually inconsistent readings, mutual exclusion is always guaranted (safety property) and that the privilege turns around the logical ring (liveness property).

\section{Safety}

Let $x_{k}(i)$ be the last value obtained by $P_{i}$ as value of $x_{k}$; it is possible to have $x_{k}(i) \neq x_{k}$ for $k \neq i$ (but $x_{i}(i)=x_{i}$ at any time).

Let us suppose that the privilege has completed $t$ full turns $(t \geq 0)$ and that in the turn $t+1$ the privilege is owned by $P_{i}$. The state variables have the following values $(\forall t \geq 0, \forall i)$ :

$$
x_{0}=\frac{a_{0}^{t+1}}{a_{1}^{t+1}}, x_{1}=\frac{a_{1}^{t+2}}{a_{2}^{t+1}}, \ldots x_{i-1}=\frac{a_{i-1}^{t+2}}{a_{i}^{t+1}}, x_{i}=\frac{a_{i}^{t+1}}{a_{i+1}^{t}}, \ldots x_{n-1}=\frac{a_{n-1}^{t+1}}{a_{0}^{t}}
$$


Let us consider $P_{m}(m \neq i)$ and suppose $P_{m}$ can obtain the privilege, ie:

$$
\prod_{k=0}^{n-1} x_{k}(m)=Q / a_{m}
$$

with:

$$
\begin{aligned}
& \text { 1) } x_{0}(m)=\frac{a_{0}^{\alpha_{0}}}{a_{m}^{\alpha_{0}}}, x_{1}(m)=\frac{a_{1}^{\alpha_{1}+1}}{a_{2}^{\alpha_{1}}}, \ldots x_{m-1}(m)=\frac{a_{m-1}^{a_{m-1}+1}}{a_{m}^{\alpha_{m-1}}} \\
& \text { 2) } x_{m}(m)=x_{m}=\frac{a_{m}^{t+2}}{a_{m+1}^{l+1}} \text { if } m<i, \frac{a_{m}^{t+1}}{a_{m+1}^{l}} \text { if } m>i \\
& \text { 3) } x_{m+1}(m)=\frac{a_{m+1}^{a_{m+1}}}{a_{m+2}^{\alpha_{m+1}}}, \ldots x_{n-1}(m)=\frac{a_{n-1}^{\alpha_{n-1}+1}}{a_{0}^{\alpha_{n-1}}}
\end{aligned}
$$

the exponents are such that:

$$
\begin{gathered}
0 \leq \alpha_{j} \leq t+1 \text { for } 0 \leq j \leq i-1 \\
0 \leq \alpha_{j} \leq t \text { for } i \leq j \leq n-1 \\
\alpha_{m}=t+1 \text { if } m<i, t \text { if } m>i
\end{gathered}
$$

Two cases have to be examined according to the case $m<i$ or $m>i$. We consider the first one.

$$
\text { (1) } \wedge m<i \Rightarrow \frac{a_{0}^{\alpha_{0}}}{a_{1}^{\alpha_{0}}} \ldots \frac{a_{m-1}^{\alpha_{m-1}+1}}{a_{m}^{\alpha_{m-1}}} \ldots \frac{a_{m}^{t+2}}{a_{m+1}^{t+1}} \ldots \frac{a_{m+1}^{\alpha_{m+1}+1}}{a_{m+2}^{\alpha_{1}+1}} \ldots=a_{0} \ldots a_{m-1} a_{m+1} . . a_{n-1}
$$

that is to say:

$$
a_{0}^{\alpha_{0}} a_{1}^{\alpha_{1}+1} \ldots a_{m-1}^{\alpha_{m-1}+1} a_{m}^{t+2} \ldots a_{n-1}^{\alpha_{n-1}+1}=a_{0}^{\alpha_{n-1}+1} a_{1}^{\alpha_{0}+1} \ldots a_{m}^{\alpha_{m-1}} \ldots a_{m+1}^{t+2} \ldots a_{n-1}^{\alpha_{n-2+1}}
$$

Moreover:

$$
m<i \wedge(2) \Rightarrow \alpha_{m-1} \leq t+1 \text { ie } \alpha_{m-1}<t+2
$$

$(2) \wedge(3) \wedge(4) \Rightarrow a_{m}^{t+2-\alpha_{m-1}}$ divides $a_{0}^{\alpha_{n-1}+1} \ldots a_{m-1}^{\alpha_{m-2+1}} a_{m+1}^{t+2} \ldots a_{n-1}^{\alpha_{n-2+1}}$

A simular reasonning hold for the case $m>i$.

Summing up:

$$
\forall m \neq i: \prod x_{k}(m)=Q / a_{m} \Rightarrow a_{m} \text { divides a product of } a_{0} . . a_{m-1} a_{m+1} . . a_{n-1}
$$

Consequently as the $a_{k}$ are prime two by two $P_{m}$ cannot enter the critical section if it is the $P_{i}$ 's turn. 


\section{Liveness}

If it is the $P_{i}$ 's turn to enter the critical section it will enter it as soon as it has received the last values of the variable $x_{m}$. (Recall the delays are finite).

\section{A TERMINATION DETECTION ALGORITHM}

\section{$4.1 \quad$ Hypotheses}

The termination detection problem is a paradigm of the distributed control [FRA80, CL85, MAT87, LY87]. A process $P_{i}$ can become passive at any time; a passive process can only be made active by receiving a message; an active process can send messages to its neigbhours. We suppose without loss of generality that a process is passive when it receives a message and that a process does not send messages to itself. The communication network is totally connected.

Detect termination consists in detecting a state of the distributed application in which all the processes are passive and all the channels are empty.

\subsection{The algorithm}

Conceptually an observer OBS is in charge of the termination detection. It can be implemented in several ways: it can be a particular site, it can be duplicated on several sites, it can be implemented by a circulating memory like a token travelling along a ring, a tree, etc [MAT87, HJPR87, RAY88].

At a conceptual level the implementation of the observer does not matter. The only hypothese we assume is that control messages OBS receives from each process are received in their sending order.

When a process $P_{i}$ becomes passive it sends to OBS a control message. Let us suppose that, when it was active for the last time, $P_{i}$ sent $p$ application messages to $P_{j}$ and $q$ to $P_{k}$. Becoming passive it scnds OBS the following control message:

$$
\operatorname{ctl}\left(a_{j}^{p} * a_{k}^{q} / a_{i}\right)
$$

The observer OBS in endowed with a variable $t$. It is initialized to the following value:

$$
t \text { init to } \prod_{i \in I} a_{i}
$$


(Where $I$ is the set of initially active processes). When it receives a control message $c t l(n / d)$ the observer OBS updates $t$ in the following way:

$$
t:=t * n / d
$$

When $t=1$ the observer claims the application is terminated: all the processes are passive and the channels are empty.

\subsection{Proof}

The proof consists in showing:

- that if the application is terminated then eventually the observer will detect it (liveness),

- and that if the termination is detected then the application is actually terminated (safety).

\section{Liveness}

When the application is terminated on the one hand each message $m$ sent to a $P_{i}$ has been received and processed, and on the other hand all the processes are passive. Consequently if $q$ messages have been sent to $P_{i}$ OBS will receive $a_{i}^{q}$ in total (by control messages) as the senders of these messages are passive. When $P_{i}$ became definitively passive (after having received these $q$ messages) it has sent the observer in total $1 / a_{i}^{q+1}$ if it was initially active (or $1 / a_{i}^{q}$ if it was initialy passive). It follows that $t=1$ when OBS has received all the control messages.

\section{Safety}

The value of $t$ represents some global snapshot of the system from the point of view of its activity. The global snapshot $\mathrm{S}$ (global state) associated to $t$ is the following one. The state of a process in $t$ is [LY87]:

- its local state when it sent the last control message received by OBS,

- the sets of messages it has sent to each of its neigbhours and the sets of messages it has received from them up to the last control message it sent and which has been received by OBS.

So at any time (each "*" stands for any non negative integer):

$$
\frac{a_{0}^{*} a_{1}^{*} \ldots a_{i-1}^{*} a_{i+1}^{*} \ldots a_{n-1}^{*}}{a_{i}^{*}}
$$


represents the values sent by $P_{i}$ and received (in fifo order) by OBS since the beginning; consequently the value of $t$ has the following form:

$$
t=\frac{a_{1}^{*} \ldots a_{n-1}^{*}}{a_{0}^{*}} \ldots \frac{a_{0}^{*} a_{1}^{*} \ldots a_{i-1}^{*} a_{i+1}^{*} \ldots a_{n-1}^{*}}{a_{i}^{*}} \ldots \frac{a_{0}^{*} \ldots a_{n-2}^{*}}{a_{n-1}^{*}}
$$

namely:

$$
t=\frac{a_{0}^{n_{0}} a_{1}^{n-1} \ldots a_{n-1}^{n_{n-1}}}{a_{0}^{d_{0}} a_{1}^{d_{1}} \ldots a_{n-1}^{d_{n-1}}}
$$

1. if $t=1$ and $\exists m$ such that $n_{m} \neq d_{m}$ then:

if $n_{m}>d_{m}$ then $a_{m}^{n_{m}-d_{m}}$ divides $a_{0}^{d_{o}} \ldots a_{m-1}^{n_{m-1}} a_{m+1}^{d_{m+1}} \ldots a_{n-1}^{d_{n-1}}$

if $n_{m}<d_{m}$ then $a_{m}^{d_{m}-n_{m}}$ divides $a_{0}^{n_{0}} \ldots a_{m-1}^{n_{m-1}} a_{m+1}^{n_{m+1}} \ldots a_{n-1}^{n_{n-1}} ! 1$

both case are impossible as the $a_{i}$ are prime. Consequently if $t=1$ then $n_{m}=d_{m}, \forall m$. In others words, to OBS's knowledge, the number of messages sent to each process $P_{i}$ equals the number of messages $P_{i}$ received.

2. Let us consider $t=1, \forall m: n_{m}=d_{m}$ and the application is not terminated. We consider two cases according to the snapshot $S$ associated to $t$ is consistent or not [LY87].

(a) The snapshot $\mathrm{S}$ is consistent (namely there is no messages received and not sent in S). As for OBS $t$ indicates the number of messages sent equals the number of messages received and as $t$ is consistent it follows that there is no message sent and not received. Consequently as the last time a process sent OBS a control message it was passive the application is terminated.

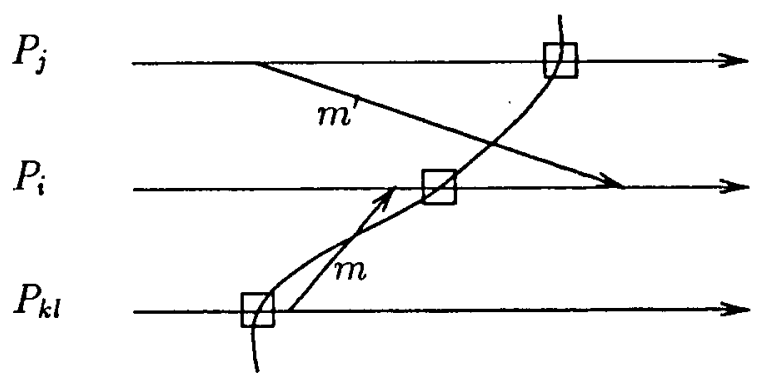

Figure 1: an inconsistent snapshot with $t=1$ 
(b) The snapshot $\mathrm{S}$ associated to $t$ is not consistent (there are messages in $\mathrm{S}$ which are perceived as received and not sent). As $t=1$ and the snapshot associated is not consistent there is (at least) one message $m$ whose reception by $P_{i}$ is known by OBS but not its sending, and there is a corresponding message $m^{\prime}$ whose sending to $P_{i}$ by some $P_{j}$ is known by OBS but not its reception. (see fig. $1, \square$ stands for the last sendings of control messages).
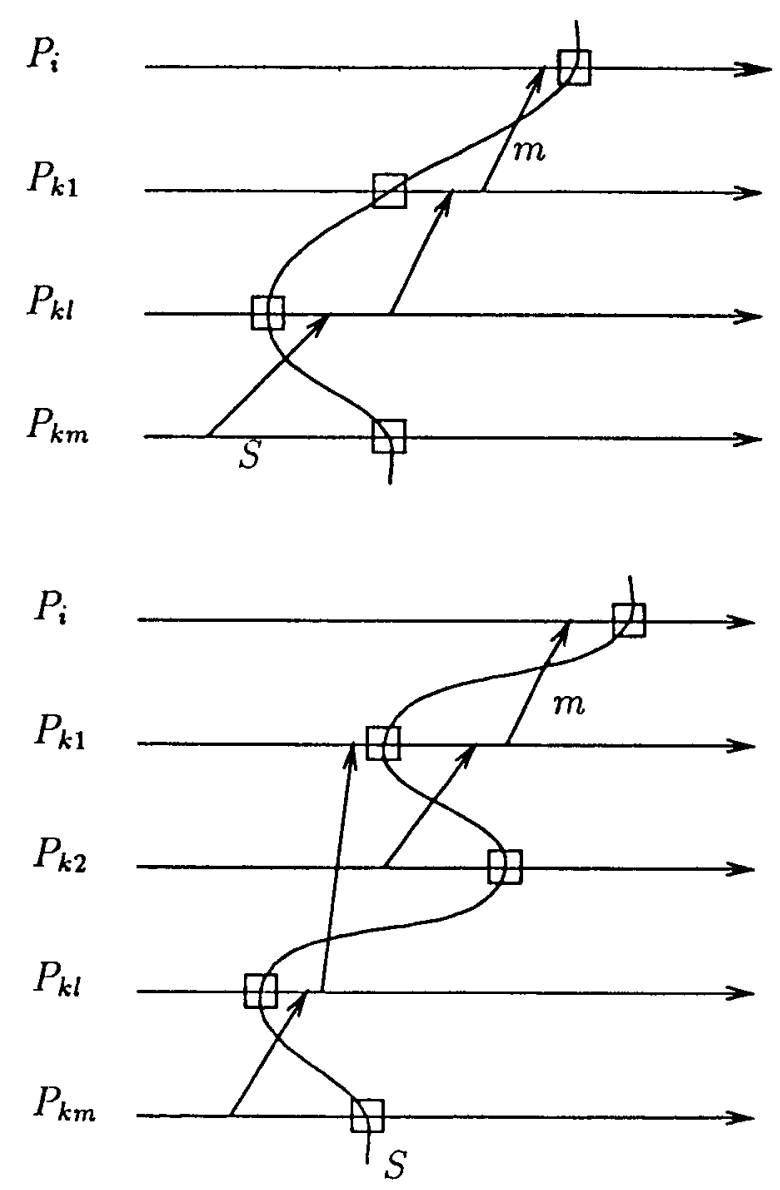

Figure 2: two inconsistent snapshots.

the message $m$ has been sent by some $P_{k 1}$ after the snapshot associated to $t$. So there is a chain of activation: $P_{k 1}$ has been activated after $S$ by $P_{k 2}$ which has been 
activated by $P_{k 3}$ after $S$ etc... such a chain is necessarily finite (the number of process is finite and an active process sends a finite number of messages): so $P_{k l}$ has been activated after the snapshot by a message sent by $P_{k m}$ before the snapshot (figure 2). Consequently there is a process $P_{k l}$ for which $n_{k l} \neq d_{k l}$ in $t$, which contradicts the hypotheses. Therefore it is not possible to have $t=1$ when there is some activity in the system.

Remark: A similar reasonning in used by Lai and Yang in [LY87] to show that inconsistent snapshot can be used to detect deadlock.

\section{ABOUT IMPLEMENTATIONS}

An implementation using vectors of dimension $n$ is obviously well-suited. For example in the termination detection case the $j^{\text {th }}$ entry of a vector sent by $P_{i}$ in a control message corresponds to the exponent of $a_{j}$. If we consider such a coding for the termination detection algorithm and an observer implemented by a token travelling along a ring (which visits periodically each process), we obtain the algorithm called "vector algorithm" proposed by Matterm in [MAT87]; if the coding uses matrices instead of vectors and if observers are put on an arbitrary number of sites we obtain an algorithm similar to the one proposed in [HJPR87].

\section{CONCLUSION}

A possible use of prime number to design distributed algorithms has been investigated. One of the major drawbacks in designing such algorithms lies in the inability for one or several processes (or for an external observer) to catch instantaneously a global state of a distributed system [CL85]. Properties of prime numbers (namely unicity of the decomposition of an integer) can allow to solve some of the problems such an inability has given rise to. Two very different distributed algorithms have been produced as an illustration of the proposed tool (one implementing a service mutual exclusion -, the other implementing an observation - termination detection -). 


\section{Acknowledgements}

This work has been partly supported by French National Project $C^{3}$ (CNRS Project on Parallelism). R. PEDRONO and A. COUVERT are acknowledged for their comments which improve on the proofs.

\section{References}

[CL85] CHANDY M.,LAMPORT L., Distributed Snapshots: determining global states in distributed systems, ACM TOCS, Vol. 3.1, (1985), pp. 63-75.

[DIJ74] DIJKSTRA E.W.D., Self-stabilizing Systems in Spite of Distributed Control, Comm. ACM, Vol. 17,11, (Nov. 1974), pp 643-644

[FRA80] FRANCEZ N., Distributed Termination, ACM Toplas, Vol. 2,1, (Jan. 1980), pp $42-55$

[HJPR87] HELARY J.M., JARD C., PLOUZEAU N., RAYNAL M., Detection of Stable Properties in Distributed Applications, Proc. 6th ACM Symposium on PODC, Vancouver, (Aug. 1987), pp 125-136

[LEL77] LE LANN G., Distributed Systems: Towards a Formal Approach, IFIP Congress, Toronto, (Aug. 1977), pp 155-160

[LAM78] LAMPORT L, Time, Clocks and the Ordering of Events in a Distributed system, Comm. ACM, Vol. 21,7, (July 1978), pp 558-565

[LY87] LAI T.H., YANG T.H., On Distributed Snapshots, Inf. Processing Letters, Vol 25, (1987), pp 153-158

[MAT87] MATTERN F., Algorithms for Distributed Termination, Distributed Computing, Vol. 2, (1987), pp 161-175

[MIS83] MISRA J., Detecting Termination of Distributed Computation using Markers, Proc. 2d ACM Symposium on PODC, Montreal, (1983), pp 290-294

[RAY88] RAYNAL M., Networks and Distributed Computation: Concepts, Tools and Algorithms, The MIT Press, (1988), 166p 
PI 452 LANCER DE RAYON : APPROCHES PARALLELES

Didier BADOUEL, François BODIN, Thierry PRIOL

16 Pages, Janvier 1989.

PI 453 UN COMPILATEUR ESTELLE MULTI-PROCESSEURS POUR L'EXPERIMENTATION D'ALGORITHMES DISTRIBUES SUR MACHINES PARALLELES

Jean-Marc JEZEQUEL, Claude JARD

54 Pages, Janvier 1989.

PI 454 REALISATION ET CALIBRATION D'UN SYSTEME EXPERIMENTAL DE VISION COMPOSE D'UNE CAMERA MOBILE EMBARQUEE SUR UN ROBOT-MANIPULATEUR

François CHAUMETTE, Patrick RIVES

36 Pages, Février 1989.

PI 455 ARCHITECTURE SYSTOLIQUE POUR LA CORRECTION

AUTOMATIQUE DE LIBELLE D'ADRESSE

Dominique LAVENIER, Jean-Luc SCHARBARG, Patrice FRISON

22 Pages, Février 1989.

PI 456 DISTRIBUTION OF OPERATIONAL TIMES IN FAULT-TOLERANT SYSTEMS MODELED BY SEMI-MARKOV REWARD PROCESSES

Gerardo RUBINO, Bruno SERICOLA

10 Pages, Février 1989.

PI 457 TANDEM QUEUES WITH FEEDFORWARD FLOWS

Kamel SISMAIL

16 Pages, Février 1989.

PI 458 PRIME NUMBERS AS A TOOL TO DESIGN DISTRIBUTED ALGORITHMS

Michel RAYNAL

14 Pages, Février 1989. 
3 